\title{
A RESTAURAÇÃO ECOLÓGICA EM DESTAQUE: UM RETRATO DOS ÚLTIMOS VINTE E OITO ANOS DE PUBLICAÇÕES NA ÁREA
}

\author{
Renata Evangelista de Oliveira ${ }^{1, *} \&$ Vera Lex Engel ${ }^{2}$ \\ ${ }^{1}$ Universidade Estadual Paulista (UNESP), Faculdade de Ciências Agronômicas - Curso de Pós Graduação (Doutorado) em Ciência Florestal, Rua José \\ Barbosa de Barros, nº 1780, Caixa Postal: 237, CEP:18610-307, Botucatu, São Paulo, Brasil. \\ ${ }^{2}$ Universidade Estadual Paulista (UNESP), Faculdade de Ciências Agronômicas - Departamento de Recursos Naturais - Ciências Florestais. \\ E-mails: reolivei@fca.unesp.br, veralex@fca.unesp.br
}

\section{RESUMO}

A partir de análise bibliográfica, são descritos aqui os principais aspectos abordados por publicações, referentes à restauração ecológica em ecossistemas diversos, nos últimos vinte e oito anos. São descritos os principais conceitos, principais temas de pesquisa e ecossistemas enfocados.

Palavras-chave: Restauração ecológica; restauração de ecossistemas florestais; conceitos e evolução.

\section{ABSTRACT}

ECOLOGICAL RESTORATION: ANALYSING THE LAST TWENTY-EIGHT YEARS OF PUBLICATIONS IN THIS AREA. The main aspects of restoration, in various ecosystems, are here discussed, by means of a bibliographic analysis of the last twenty-eight years. The main concepts, research themes and target ecosystems are also described.

Keywords: Ecological restoration; forest ecosystems restoration; concepts and evolution.

\section{RESUMEN}

RESTAURACIÓN ECOLOGICA: ANALISIS DE LOS ULTIMOS VENTIOCHO AÑOS DE PUBLICACIONES EN ESTA AREA. A partir del análisis bibliográfico de los últimos 28 años, se describen los aspectos más importantes planteados en las publicaciones relacionadas a la restauración ecológica en diversos ecosistemas. Se describen los principales conceptos, temas de investigación y ecosistemas blanco.

Palabras claves: Restauración ecológica; restauración de ecosistemas forestales; conceptos y evolución.

\section{INTRODUÇÃO}

A ecologia da restauração tem tido avanços significativos nas últimas décadas, contribuindo para as práticas de recuperação de ecossistemas degradados e para o desenvolvimento e ampliação do pensamento ecológico. Essa área da ecologia vem se tornando uma ferramenta importante para o manejo, conservação e recuperação de muitos ecossistemas ao redor do mundo.

A restauração ecológica, aqui definida como 'o processo de assistência à recuperação de um ecossistema que foi degradado, danificado ou destruído' (SER 2004), tem como base científica a ecologia da restauração, e pode ser descrita como um conjunto de práticas que compõem o campo da restauração, incluindo aspectos políticos, tecnológicos, econômicos, sociais e culturais (Higgs 2005, Hobbs 2005). É encarada de várias formas por diferentes autores, seja como um campo de atuação, uma arte, uma disciplina, uma sub-área da ecologia e também como prática, ou mesmo como uma ciência aplicada. Entretanto, projetos de restauração ecológica com embasamento científico se distinguem 
dos demais pela incorporação de conhecimento da teoria ecológica e da aplicação do método científico (Palmer et al. 2006).

O levantamento e análise dos trabalhos que vêm sendo desenvolvidos e publicados no campo da restauração ecológica pode auxiliar na avaliação do grau de maturidade e nível de consolidação dessa área do conhecimento. Essa avaliação pode estar baseada em um levantamento e sistematização de seus pressupostos teóricos, demandas e objetivos, métodos, técnicas, lacunas, etc, e também na caracterização das pesquisas realizadas, considerando-se diferentes ecossistemas (Oliveira \& Engel 2009).

Neste trabalho, fizemos uma análise bibliográfica, com ênfase na percepção dos rumos da restauração ecológica, a partir de uma perspectiva temporal e baseada nas discussões científicas e publicações na área. Foi analisada a construção gradual dos conceitos relativos à restauração ecológica e a incorporação de aspectos técnicos, ecológicos, sociais e culturais na construção desses conceitos, com síntese do conteúdo científico acumulado nos últimos vinte e oitos anos, tendo a restauração ecológica como assunto principal.

\section{MATERIAL E MÉTODOS}

\section{AS PUBLICAÇÕES NA ÁREA DA RESTAURAÇÃO ECOLÓGICA: CARACTERIZAÇÃO E TRAJETÓRIA}

Para caracterização das publicações e observação dos rumos das pesquisas em restauração ecológica, foi feito levantamento dos artigos científicos publicados em inglês que apresentassem em seus títulos o termo restoration, utilizando a base bibliográfica ISI Web of Knowledge. O intervalo de tempo analisado foi de vinte e oitos anos, de 1980 a 2008. Para incluir artigos que se relacionassem diretamente à discussão sobre a restauração ecológica, ou à descrição de projetos de restauração em diferentes ecossistemas, optouse pela seleção de 13 periódicos da área ecológica/ biológica disponíveis na base utilizada: Restoration Ecology; Ecological Engineering; Conservation Biology; Biodiversity and Conservation; Journal of Applied Ecology; Forest Ecology and Management; Environmental Management; Science; Applied Vegetation Science; Biotropica; Oecologia; Landscape Ecology e Ecology.
Todos os artigos foram incluídos numa lista para verificação e, a partir dos seus títulos, foi feita uma primeira triagem, de forma a excluir os artigos que não se referiam à restauração ecológica ou restauração de ecossistemas. Dessa forma, foram excluídos artigos cujo enfoque do termo restauração não condizia com aquele proposto para esta pesquisa. $\mathrm{O}$ conceito de restauração ecológica aqui adotado foi aquele preconizado pela Society of Ecological Restoration (SER 2004).

Os artigos selecionados foram categorizados, inicialmente, em artigos teóricos ou conceituais (AT) e estudos de caso (EC) (como em Petenon \& Pivello 2008). Esses artigos foram ordenados no tempo - a partir do ano de publicação, e foi feita a contabilização de artigos por periódico. Os estudos de caso foram divididos em três categorias: estudos de caso em "restauração", com enfoque na restauração ecológica, "recuperação de áreas degradadas" e "remediação ou biorremediação".

$\mathrm{O}$ enfoque dos estudos de caso (ECs) em restauração foi investigado a partir da avaliação dos títulos dos mesmos, a partir da busca por termos específicos (como por exemplo: evaluation, success, indicators, soil, forest, policies ou political, social, participation, alien ou exotic species, etc).

\section{O ENFOQUE DA RESTAURAÇÃO ECOLÓGICA E SEUS TEMAS-CHAVE}

A análise da ciência ou área da restauração ecológica, a partir de uma perspectiva temporal, foi considerada interessante para compreender os rumos dessa área do conhecimento nas últimas décadas. Complementarmente à análise dos artigos publicados, essa análise incluiu uma descrição do que se compreende por restauração ecológica, com os principais avanços e tendências, no período definido para essa pesquisa. Foi feita uma coletânea, de diferentes conceitos e definições, publicados para a restauração ecológica, desde 1980, e avaliação, no tempo, de suas principais abordagens e enfoques. Para complementar a descrição e avaliação dos conteúdos publicados, foi feito um levantamento das palavraschave mais utilizadas, nos artigos categorizados como artigos teóricos, por década analisada. Para isso, foram levantadas as palavras-chave utilizadas pelos autores, e levantado o número de vezes em que 
as mesmas foram citadas, assim como o número de citações por década analisada.

\section{O DESENVOLVIMENTO DE PESQUISAS E AÇÕES DE RESTAURAÇÃO EM DIFERENTES ECOSSISTEMAS}

Os artigos categorizados como estudos de caso em restauração foram checados, ainda, quanto ao registro do tipo e localização (ocorrência) do ecossistemaalvo. Os ecossistemas restaurados ou em processo de restauração descritos foram agrupados em: (i) campos, pradarias, e outros ecossistemas dominados por gramíneas e herbáceas; (ii) ecossistemas florestais (florestas nativas e plantações florestais); (iii) áreas úmidas; (iv) ecossistemas ripários (restauração de rios, riachos, canais e vegetação ripária); (v) lagos e (vi) outros.

No caso dos ecossistemas florestais, foram listados todos os artigos categorizados como estudos de caso que abordaram a restauração de ecossistemas florestais em vários países. Destes, foram selecionados os artigos que apresentaram ou discutiram ações de restauração realizadas a fim de suplantar barreiras específicas advindas do processo de degradação, ou aqueles que apontaram enfoques específicos para a necessidade de restauração.

A partir dos abstracts dos mesmos, foram checadas as práticas e ações de restauração apontadas (ou mesmo citadas) pelos autores, que foram contabilizadas segundo categorias de filtros ambientais (propostos por Hobbs \& Norton 2004 e Nuttle 2007). Foram considerados os filtros: estrutura da paisagem, interações ecológicas (abrigando competição, herbivoria, mutualismo, polinização e dispersão), dispersão/propagação, distúrbios, ordem de chegada de espécies, legado ambiental e pool de espécies.

\section{RESULTADOS E DISCUSSÃO}

\section{AS PUBLICAÇÕES NA ÁREA DA RESTAURAÇÃO ECOLÓGICA: CARACTERIZAÇÃO E TRAJETÓRIA}

O primeiro levantamento levou a uma listagem de mais de 700 artigos, que após uma primeira triagem a partir somente dos títulos, resultaram em 672 artigos.
Uma segunda análise a partir da conferência dos títulos e abstracts levou a 617 artigos científicos que se referiram ao tema 'restauração ecológica', no período considerado. Desses, 78 (13\%) foram considerados artigos teóricos ou conceituais, e 539 (87\%) estudos de caso. Dos estudos de caso, 496 artigos (92\%) referiram-se à restauração ecológica de ecossistemas propriamente dita, 37 (7\%) à descrição de projetos de recuperação de áreas degradadas (áreas mineradas, depósitos, áreas de empréstimo, etc), e seis $(1 \%)$ de remediação ou biorremediação.

Tabela 1: Número de artigos analisados (ECs - estudos de caso; ATs artigos teóricos) por periódico

Table 1. Number of papers analyzed (ECs - case studies; ATs - Theoretical studies) by journal title.

\begin{tabular}{lcc}
\multicolumn{1}{c}{ Periódico } & No de ECs & $\begin{array}{c}\mathbf{N}^{\mathbf{0}} \mathbf{d e} \\
\text { ATs }\end{array}$ \\
\hline $\begin{array}{l}\text { Journal of Applied Ecology } \\
\text { Environmental Management }\end{array}$ & 61 & 5 \\
Forest Ecology and & 42 & 8 \\
Management & 44 & 0 \\
Oecologia & 01 & 0 \\
Applied Vegetation Science & 29 & 2 \\
Biodiversity and Conservation & 18 & 1 \\
Ecological Engineering & 47 & 1 \\
Conservation Biology & 14 & 8 \\
Restoration Ecolology & 236 & 53 \\
Biotropica & 1 & 0 \\
Landscape Ecology & 5 & 0 \\
\hline
\end{tabular}

A maior parte dos trabalhos publicados sobre o tema foi da revista Restoration Ecology, seguidos de Journal of Applied Ecology, Envioronmental Management e Forest Ecology and Management (Tabela 1). Destaca-se a grande influência do periódico Restoration Ecology, com o número mais expressivo de trabalhos (289 no total, no período analisado), já que é específico na área da restauração ecológica e ecologia da restauração.

De 1980 a 1989 foram publicados seis artigos (um teórico- AT- e cinco estudos de caso - ECs); 127 artigos de 1990 a 1999 (28 ATs e 99 ECs), e 441 de 2000 a 2008 (sendo 49 ATs e 392 ECs) (Figura 1), ficando claro um grande aumento no tempo de artigos publicados, demonstrando gradativa ampliação do 
interesse na área da restauração. A grande maioria dos estudos de caso analisados discute, apresenta ou descreve ações e métodos de restauração para ecossistemas específicos. Como afirma Allen (2003), então editora-chefe da Restoration Ecology, 80\% dos artigos publicados na revista, até essa data, referiramse a "experimentos de restauração ou observações em sítios restaurados".



Figura 1. Número de artigos publicados em restauração ecológica, nas revistas Journal of Applied Ecology; Environmental Management; Forest Ecology and Management; Oecologia; Applied Vegetation Science; Biodiversity and Conservation; Ecological Engineering; Conservation Biology; Restoration Ecolology; Biotropica; Landscape Ecology ano a ano, no período de 1980 a 2008.

Figure 1. Number of papers published in ecological restoration in journals Journal of Applied Ecology; Environmental Management; Forest Ecology and Management; Oecologia; Applied Vegetation Science; Biodiversity and Conservation;Ecological Engineering; Conservation Biology; Restoration Ecolology; Biotropica; Landscape Ecology, from 1980 to 2008.

A análise dos títulos dos artigos categorizados como estudos de caso auxilia na descrição dos principais enfoques e abordagem das publicações. Dos estudos de caso, 20 referiram-se à ecologia de paisagens e sua relação com a restauração, ou apresentaram projetos de restauração no contexto de paisagens (de regiões temperadas ou tropicais, urbanas ou rurais). Esses artigos, no geral, abordaram características da paisagem em que se insere um determinado ecossistema a restaurar, aspectos históricos e de alteração da paisagem antes e depois da restauração, ou enfocaram a restauração analisando-se a paisagem enquanto escala, e não abordaram um ecossistema específico. Numa análise mais aprofundada desses trabalhos, complementada por um levantamento mais específico voltado à restauração de e em paisagens, verificou-se que os enfoques principais foram a documentação de paisagens históricas e de mudança do uso do solo, e a discussão de possíveis estratégias de restauração voltadas a populações animais específicas - existe grande enfoque na ecologia de paisagens voltado à reconstrução e/ou análise de habitats para fauna. Poucos trabalhos referiram-se a espécies exóticas e/ ou invasoras e seu papel em paisagens específicas. Somente $10 \%$ dos trabalhos analisados incluem ou mencionam aspectos sócio-econômicos ou o papel e/ou importância das populações locais e sua participação na restauração de paisagens (como analisado por Oliveira et al. 2009).

O componente solo, na restauração ecológica, é abordado em aproximadamente $10 \%$ dos artigos analisados (50 no total). Já aspectos referentes à sucessão, e processos sucessionais na restauração estão destacados em 15 artigos (3\%). A avaliação do sucesso da restauração, aliada ao uso de indicadores, esteve presente em $12 \%$ dos estudos de caso descritos. Já o enfoque voltado às espécies invasoras, exóticas ou indesejáveis teve pouco destaque, estando presente nos títulos de pouco mais de $1,5 \%$ dos trabalhos. Não foram encontrados títulos referentes à participação social, ou a aspectos políticos ou sociais na restauração, no período considerado, para o conjunto de dados analisado. 
O ENFOQUE DA RESTAURAÇÃO ECOLÓGICA E SEUS TEMAS-CHAVE

\section{A evolução do conceito de "restauração ecológica"}

Foram levantados vários conceitos e definições, publicados e discutidos para a restauração ecológica, desde a década de 80 até o tempo presente (Anexo 1). Percebe-se, com base neste levantamento, que não existe um conceito unificado e completamente aceito para a Ecologia da Restauração, ou para a Restauração Ecológica, assim como ocorre para quaisquer outras áreas, ciências ou linhas de pensamento.

Por exemplo, percebe-se uma clara sobreposição entre o que se entende por 'reabilitação' ' 'restauração' de ecossistemas. Ou seja, muitas definições dadas ao termo 'reabilitação' contêm os mesmos elementos e objetivos conferidos atualmente à 'restauração ecológica' (Aronson 1993 citado em Walker \& Del Moral 2003, ITTO 2002, Lamb \& Gilmour 2003). As estratégias descritas por ITTO (2002), por exemplo, para acelerar a reabilitação de áreas florestais degradadas são as mesmas citadas por outros autores, que usam o termo 'restauração' como o plantio de mudas florestais nativas, plantio de mudas florestais não nativas como pioneiras, utilização de árvores remanescentes ou plantio de módulos arbóreos, semeadura de espécies arbustivas (Lamb et al., 1997, entre outros). E, em alguns casos, alguns autores preferem utilizar o termo 'reabilitação' para as ações com objetivo de recuperar características referentes à estrutura e função de um determinado ecossistema, e preferem a utilização do termo 'restauração' para um processo onde o ecossistema original tem grande influência ou o objetivo final pretendido é algo muito próximo do mesmo (Lamb et al 1997, Walker \& Del Moral 2003, Lamb \& Gilmour 2003). Apesar de toda a polêmica, segundo Atkinson (1994), os significados originais dos termos, no latim, são idênticos.

A maioria dos conceitos listados relaciona a restauração ecológica a ecossistemas degradados. A utilização do termo 'ecossistema', mais do que o de comunidade, ou mesmo paisagem, talvez reflita a compreensão de que a restauração está relacionada a todo um conjunto de elementos bióticos e abióticos e às interações entre esses elementos, entradas e saídas de energia, fluxos e processos diversos, etc, não deixando de lado características estruturais e de composição, mas se referindo também ao funcionamento, com um enfoque mais dinâmico e processual, envolvendo diferentes trajetórias.

O conceito ecológico de ecossistema refere-se a um conjunto de organismos que interagem com o ambiente físico, incluindo a matéria e energia que podem assimilar, num determinado local. Essa definição inclui o reconhecimento de fluxos de energia e matéria, e também da evolução dos componentes do sistema, sua trajetória histórica, as interações e regras de organização entre organismos, comportamento e persistência de populações, e informações na genética e outras estruturas do ecossistema (Parker \& Pickett 2000). Não existem limites físicos definidos para os ecossistemas, porque sua distribuição ao longo do planeta é relativamente contínua. Os diferentes ecossistemas são reconhecidos, arbitrariamente, por sua localização e composição de espécies, e apresentam dois atributos principais - sua estrutura e função - formadas por diferentes elementos, e que podem ser utilizados para ilustrar os danos que um determinado ecossistema pode sofrer. Sua estrutura e função podem ser reduzidas, drástica ou continuamente, com a degradação (Bradshaw 2002).

Para Ehrenfeld \& Toth (1997), 'ecossistema' é, ao mesmo tempo, um conceito difuso e a mais forte idéia de organização que pode ser usada na restauração. A palavra 'ecossistema' tem conotação de um conjunto de elementos teóricos que bem descrevem as conexões funcionais entre os organismos residentes, e entre a biota e o ambiente físico. Outro aspecto interessante levantado por esses autores é que a ecológica baseia-se na idéia de que um sítio restaurado deva ser auto-sustentável (sem demandas de inputs externos). Também para Engel \& Parrotta (2003) o princípio fundamental implícito no conceito de restauração ecológica é o da sustentabilidade, com o sítio restaurado se auto-sustentando a longo prazo e sem necessidade de intervenção ou manejo externo.

Termos como 'estrutura', 'dinâmica', 'diversidade', 'integridade ecológica', 'potencial biológico', 'sistema funcional e auto-regulado', presentes nas definições, comprovam essas colocações. Os termos 'estrutura' e 'função' estão muito presentes nas definições, e representam a idéia de uma composição mínima de elementos a serem incluídos, inseridos, ou mantidos no local alvo da restauração, e o desenvolvimento, manutenção e/ou 
garantia de funcionamento e ocorrência de processos fundamentais à sua manutenção, a longo prazo. Esta estrutura/composição e funcionamento mínimos desejados mudam de ecossistema para ecossistema, e de acordo com o nível de conservação e/ou degradação.

Contrariamente a crenças anteriores de que, basicamente, a prática da restauração estaria baseada em assumir que a natureza seria fixa e imutável (Jordan 1993, citado em Urbanska et al. 2000), a idéia de que os ecossistemas são complexos, não lineares, geralmente imprevisíveis, e que podem ocorrer num sem número de estados alternativos, advindos de diferentes históricos de degradação e de intervenção humana, vem ganhando força. E também a de que a dinâmica espacial e temporal dos ecossistemas pode a ser base fundamental para a restauração (Hobbs 2005, Parker \& Pickett 2000).

Fica claro, a partir das definições aqui elencadas, que a definição da restauração está diretamente relacionada a seus objetivos. Ehrenfeld (2000) discute a necessidade de se definirem objetivos mais realistas e aponta que sua definição é o componente mais importante de um projeto de restauração, uma vez que define as expectativas, direciona planos de ação e determina o tipo e extensão do monitoramento no futuro. Esse autor coloca que os objetivos para a restauração são relativos à restauração de espécies, às funções do ecossistema e dos serviços ambientais prestados por ele. Para Whisenant (1999) esses objetivos realistas devem considerar a extensão da degradação, o potencial ecológico do local, os usos da terra pretendidos e as restrições sócio-econômicas. E, já que os ecossistemas naturais são essencialmente dinâmicos (e não estáticos ou previsíveis) e estão em constante mudança, o processo de restauração, ao invés de definir uma determinada composição de espécies como objetivo final, deve redirecionar processos essenciais do ecossistema para uma trajetória diferenciada daquela definida pela degradação.

Durante um bom tempo, o termo 'restauração' foi utilizado num sentido restrito, significando o retorno ao estado original do ecossistema, difundindo erroneamente que os objetivos da restauração seriam praticamente impossíveis de se alcançarem. Restaurar integralmente os ecossistemas, retornando-os às suas condições originais é praticamente impossível, dadas as suas características dinâmicas. E, não há como se determinarem a estrutura e função originais, devido à total ausência de dados históricos sobre as mesmas. Tentar voltar no tempo, a um estado pré-intervenção humana, é um objetivo não realista e não factível, já que a estrutura dinâmica dos ecossistemas demonstra que suas características se alteram em escala espacial e temporal, e, dessa forma, o conceito de "estados estáveis alternativos" deixa claro que não há um estado final correto a se buscar (Engel \& Parrotta 2003, Hobbs 2005).

Em muitos trabalhos permanece a visão de que o ecossistema original deva ser a referência para o processo de restauração. Entretanto, para Choi (2007), a determinação de ecossistemas-alvo é altamente arbitrária e subjetiva, e Davis (2000) coloca que o primeiro grande dilema da restauração foi o de se utilizar como alvo os ecossistemas do passado. Mas em algumas das definições mais recentes, na década de 2000 parece haver uma tentativa de se diminuir, gradativamente, a alusão à utilização do ecossistema original (e suas características estruturais e funcionais) como o alvo, ou objetivo final da restauração. A alusão à restauração como tentativa de se 'auxiliar a recuperação', ou buscar um estado mais próximo 'quanto possível' do original, torna os objetivos mais factíveis, e, ao mesmo tempo, permite considerações e definições específicas, para diferentes ecossistemas, em diferentes condições.

De acordo com Bradshaw (2002), a definição dos objetivos da restauração é uma questão arbitrária, já que o ecossistema final pretendido não é uma entidade fixa. A aceitação do termo num sentido mais amplo traz em sua definição certa flexibilidade, em que o objetivo refere-se, por exemplo, a somente uma determinada característica ou processo específico. Segundo o autor, talvez devamos esperar pela reabilitação de certas características. Várias definições de objetivos são por demais ambiciosas, e não refletem a prática da restauração, mas talvez reflitam um conceito almejado, desejado, de que algumas características dos ecossistemas degradados fossem, realmente, plenamente recuperáveis. Por exemplo, o objetivo de 'recriar' as condições que foram destruídas (Bradshaw \& Chadwick 1980), ou de 'recriar' a estrutura e função perdidas de um ecossistema (USNRC 1992 citado em Higgs 1997), ou de se planejarem ações que levem à recuperação 
'completa', a uma condição pré-distúrbio (Aronson et al. 1993 citado em Walker \& Del Moral 2003).

Para Hobbs \& Harris (2001), os objetivos da restauração não podem ser definidos a partir de atributos estáticos. A definição de objetivos claros e factíveis é essencial, e deve enfocar as características desejadas para o ecossistema no futuro, mais do que em relação ao que este foi no passado. Choi (2004) defende um paradigma 'futurista' para a restauração, (i) em que se estabeleçam objetivos factíveis e dinâmicos para um ambiente futuro, e não passado, (ii) que assuma a possibilidade de múltiplas trajetórias, dada à natureza imprevisível de comunidades ecológicas e ecossistemas, (iii) que aborde escalas mais amplas, como as de ecossistemas e paisagens, (iv) que inclua a avaliação do progresso da restauração com critérios específicos, baseados em inferências quantitativas, e (vi) que considere a necessidade de monitoramento a longo prazo das ações de restauração. O que chama a atenção, entretanto, nas definições elencadas, é a total ausência de questões sociais, econômicas e culturais, relativas à restauração de ecossistemas degradados, que também deveriam estar incluídas na definição de seus objetivos.

O documento divulgado pela SER em 2004, que apresenta várias definições relativas ao tema da restauração, traz elencados quais seriam os atributos necessários a um ecossistema para que o mesmo seja considerado restaurado, propondo formas ou critérios para o monitoramento da restauração. Segundo a SER (2004), um ecossistema pode ser considerado restaurado quando atinge ou apresenta um determinado conjunto de atributos. Contém recursos bióticos e abióticos suficientes para continuar seu desenvolvimento sem necessidade de assistência ou subsídio externo; é capaz de se auto-sustentar estruturalmente e funcionalmente; demonstra resiliência a níveis normais de stress e distúrbios; e interage com os ecossistemas contíguos a ele, em termos de fluxos bióticos e abióticos, e no que se refere a interações culturais.

\section{Os temas-chave (ou o conteúdo) da restauração ecológica}

O levantamento das palavras-chave citadas nos artigos teóricos complementa a análise dos editoriais, no sentido de permitir a observação da evolução do interesse por temas específicos, e se houve mudanças nesse sentido no tempo. A compreensão da importância das palavras-chave baseia-se no pressuposto de que estas demonstram qual o enfoque dado pelos autores nas discussões trazidas pelos artigos por eles escritos; ou seja, mostra quais os temas considerados importantes pelos mesmos para a discussão teóricoconceitual da restauração ecológica.

Foram 78 os artigos, categorizados como teóricos ou conceituais conforme já descrito. No que se refere à observação dos temas de interesse década a década, esta é possível somente para os anos 1990 e 2000, já que somente um artigo teórico foi incluído na análise na década de 80 . Foram 39 as palavraschave analisadas. O levantamento realizado pode ser considerado relevante, porque demonstra o interesse por temas específicos, ou mesmo a inserção gradual desses temas no debate sobre a restauração ecológica com o passar do tempo.

A palavra-chave restoration, como esperado, foi a mais utilizada, estando presente em 96\% dos artigos analisados. No mais, não existem palavras-chave com enfoque diferencial ou de grande importância, em termos quantitativos, considerando sua utilização no número total de artigos analisados.

Os termos landscape, ecosystem, management foram os mais utilizados. Os números podem indicar que a abordagem da restauração considerando o contexto ou a escala da paisagem foi equivalente para as décadas de 90 e 2000. Já o interesse em ações de manejo associadas à restauração, ou ao manejo de ecossistemas como ação complementar, ou como objetivo ou mesmo método de restauração, aumentou consideravelmente na última década (Pastorok et al. 1997, Ehrenfeld 2000, Brown et al. 2004, Beckage et al. 2005, Manning et al. 2006, e outros).

A reflexão sobre a ecologia da restauração enquanto 'ciência', e sobre seus 'paradigmas', ou mesmo alterações dos mesmos surgem como temas principais somente na última década, embora a 'teoria ecológica' e sua relação com a restauração já esteja presente em dois dos artigos analisados na década de 90 (Palmer et al. 1997, Clewell \& Rieger 1997, Gillilan et al. 2005, Schaefer 2006, Cabin 2007, Giardina et al. 2007, Halle 2007, Temperton 2007, Choi 2007).

A utilização das palavras-chave 'serviços ecossistêmicos', 'custos da restauração', 'mudanças 
climáticas' confirmam a inserção desses temas de interesse também somente na última década (Holl \& Howarth 2000, Ehrenfeld 2000, Beckage et al. 2005, Crossman et al. 2007, Dorrough et al. 2008, Verhoeven et al. 2008). A preocupação com questões de "fragmentação" e "conectividade" na paisagem ganham espaço também na última década (Huxel \& Hastings 1999, Maina \& Howe 2000, Schrot et al. 2005, Verhoeven et al. 2008).

A discussão sobre o conceito de restauração, seus objetivos e diretrizes é iniciada já na década de 90 , e permanece em destaque na década seguinte (o que é demonstrado pelas palavras-chave concept, conceptual, efforts, goals, guidelines). Da mesma forma, a avaliação da restauração (evaluation) ganha destaque na década de 90 , assim como o interesse na discussão sobre o que deve ser considerado 'sucesso' na restauração. A discussão sobre thresholds ou limiares de transição, e 'filtros bióticos e abióticos', bem como suas implicações e importância para a restauração, são temas bem recentes, e só estão presentes nas discussões na década de 2000, e em poucos artigos. Questões estratégicas, referentes à 'tomada de decisão', 'planejamento da restauração' e 'participação', estão presentes desde os anos 90, mas ganham destaque na década seguinte. E a preocupação ou o interesse na abordagem 'humana' ou 'social' na restauração, ou conforme cita Allen (2003), na 'dimensão humana', ganha destaque também nos anos 2000 .

\section{O DESENVOLVIMENTO DE PESQUISAS E AÇÕES DE RESTAURAÇÃO EM DIFERENTES ECOSSISTEMAS}

Os ecossistemas mais estudados ou que apresentaram maior quantidade de referências foram os ecossistemas florestais (Figura 2), e os formados por campos, pradarias e outros, dominados por gramíneas e herbáceas ( $26 \%$ e $17 \%$, respectivamente) - cabe ressaltar que a categorização utilizando o termo 'ecossistemas florestais' refere-se aqui a florestas nativas, exóticas, plantações florestais, ou projetos envolvendo espécies florestais. A restauração ecológica desses ecossistemas foi enfocada em 130 dos artigos publicados (ou seja, estes abordam plantações florestais, florestas homogêneas ou heterogêneas, espécies florestais, reflorestamentos, etc). Os ecossistemas aquáticos perfizeram $9 \%$ dos artigos consultados, e áreas úmidas e ripárias (incluindo ou não ambientes aquáticos como alvo ou como parte do alvo da restauração), 14\% (Figura 2).

Diversos autores (Fattorini \& Halle 2004, Hobbs \& Norton 2004, Nuttle 2007) encaram as ações realizadas durante o processo de restauração do

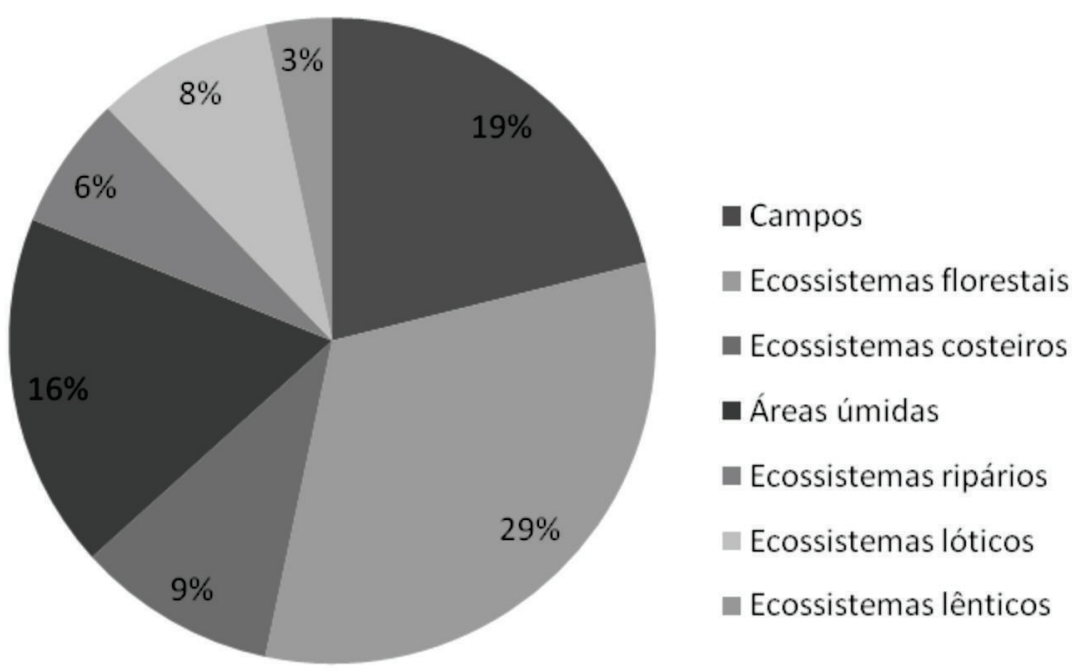

Figura 2. Ecossistemas-alvo enfocados nos estudos de caso, em porcentagens. Figure 2. Ecosystems focused in case studies, in percentages. 
ecossistema como ajustes dos filtros bióticos e abióticos a uma determinada configuração desejada. Essas ações de restauração permitem a abertura desses filtros, com o conseqüente estabelecimento de um pool de espécies na área-alvo da restauração. Foram listados 105 trabalhos, publicados de 1995 a 2008, cujas ações de restauração descritas (ou alusão à necessidade de ações) puderam ser associadas a filtros específicos. Os dados obtidos demonstram haver atenção semelhante a todos os filtros (principalmente no que se refere aos filtros bióticos) quando se pensa em ações de restauração de ecossistemas florestais, ao redor do mundo. Pode-se afirmar que existe até mesmo um equilíbrio na atenção sobre esses filtros (Figura 3), ou seja, nenhum parece ter mais importância no enfoque das ações de restauração em ecossistemas florestais quando se considera o total de trabalhos (todos são citados em aproximadamente $25 \%$ dos trabalhos analisados).

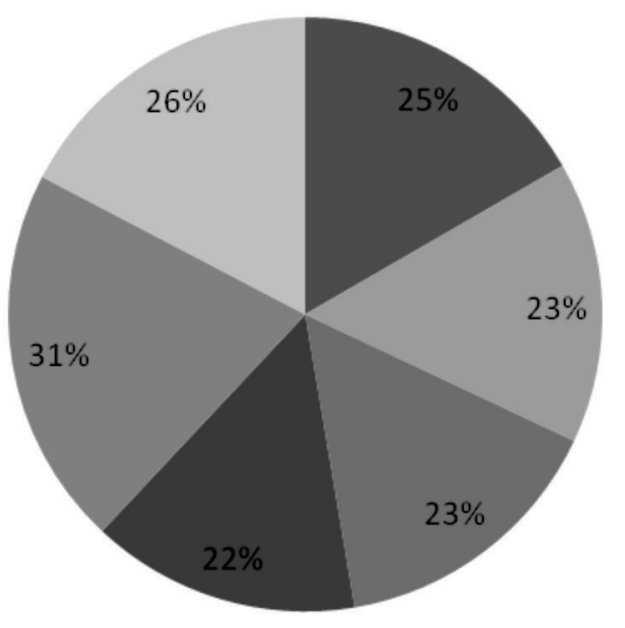

Estrutura de paisagem

- Dispersão/Propagação

Distúrbios

Ordem de chegada de espécies

Pool de espécies

- Interações ecológicas

Figura 3. Filtros bióticos e abióticos enfocados nas ações de restauração em ecossistemas florestais temperados e tropicais, no período analisado. Figure 3. Biotic and abiotic filters focused on restoration activities in temperate and tropical forest ecosystems in the analyzed period.

\section{CONSIDERAÇÕES FINAIS E CONCLUSÕES}

Whitmore (1988) afirma que um trabalho de revisão busca 'a construção de uma síntese'. Trata-se inicialmente de um trabalho especulativo, audacioso, que busca padrões gerais num determinado tema. Permite o desenvolvimento de um arcabouço de conhecimento e compreensão de um tema ou área. O trabalho de síntese e de revisão baseiase em uma quantidade de dados consistentes, e marca e/ou demonstra a maturidade de um campo de conhecimento, consolidando-o. Muitas vezes a revisão aponta lacunas e pode também, indicar caminhos e alternativas. O foco deste trabalho de revisão foi a Restauração Ecológica.

Como bem apontam Oliveira \& Engel (2009), é possível diagnosticar um caráter interdisciplinar na restauração ecológica, enquanto área do conhecimento e sub-área da ecologia, e enquanto área de atuação prática. Essa interdisciplinaridade refere-se às características relacionadas à estrutura e função dos ambientes a serem restaurados, em todos os níveis de organização ecológica (espécies, populações, comunidades, ecossistemas e paisagens), incluindo questões ecológicas, econômicas, sociais, culturais e políticas em ambientes rurais e urbanos. Os artigos analisados discutem estratégias, métodos e técnicas para vários tipos de ecossistemas (aquáticos e terrestres), sejam mais ou menos degradados, e referem-se aos vários componentes (físicos e bióticos) desses ecossistemas.

Os dados demonstram um aumento crescente no interesse pela restauração ecológica enquanto tema de pesquisa, o que é demonstrado pelo aumento com o tempo do número de artigos publicados. No que se refere aos temas de interesse, não foram encontrados temas de destaque, ao menos em termos quantitativos. O método utilizado aqui pôde apenas 
apontar o ingresso no tempo de alguns temas mais específicos. Mas de qualquer forma, parece haver um equilíbrio entre as abordagens e temas selecionados para discussão e publicação.

A avaliação temporal dos conceitos publicados não demonstra grandes alterações ou incorporação de novos aspectos, e muito menos, de grandes mudanças no que se refere a trajetórias alternativas nos ecossistemas. Não se observam mudanças efetivas no conceito, incluindo, por exemplo, uma visão mais dinâmica dos ecossistemas e na forma de se encarar o "produto final da restauração". Isso acarretaria em mudanças na forma de se planejarem suas ações, projetos, na avaliação e busca de indicadores de sucesso. Não cabe aqui dizer que isso não esteja ocorrendo, mas a listagem de conceitos e definições elencadas não demonstra ou não faz alusão a essas mudanças. Ou, talvez fique clara a demanda por uma definição, para a restauração de ecossistemas, mais condizente com a possibilidade real de se buscar a recuperação de múltiplas características, em ecossistemas degradados, e considerando diferentes trajetórias, de acordo com suas características ecológicas, com os múltiplos processos sucessionais antes e depois da degradação, e de acordo com o histórico da área em questão; e, finalmente, que incorpore ou considere sua função social, ou a sua relação com as populações humanas.

A avaliação a partir dos filtros ambientais enfocados ou descritos não permitiu, necessariamente, a comparação de ações de restauração, mas esclarece sobre quais os enfoques e objetivos, que são (ou devem ser) levados em conta para o planejamento das ações em diferentes ecossistemas florestais, temperados e tropicais.

Cabe ressaltar que, no geral, percebe-se uma inclusão muito incipiente da dimensão humana, política, social e econômica nas ações e na temática da restauração, e o que precisaria ser incorporado urgentemente, tanto nas discussões teóricas e conceituais como na prática da restauração.

AGRADECIMENTOS: Este trabalho é parte de uma pesquisa intitulada "O estado da arte da ecologia da restauração e sua relação com a restauração de ecossistemas florestais no Bioma Mata Atlântica", financiada pela FAPESP - Fundação de Amparo à Pesquisa do Estado de São Paulo.

\section{REFERÊNCIAS}

ALLEN, E. 2003. New directions and growth of Restoration Ecology. Restoration Ecology, 11: 1-2.

ATKINSON, I.A.E. 1994. Guidelines to the development and monitoring of Ecological Restoration programmes. Wellington, New Zealand, Department of Conservation Technical Series, 7, 34 p. <http://www.doc.govt.nz/upload/documents/science-andtechnical/docts07.pdf>. (Acesso em 12/12/ 2008).

BECKAGE, B.; PLATT, W.J. \& PANKO, B. A. 2005. Climatebased approach to the restoration of fire-dependent ecosystems. Restoration Ecology, 13: 429-431.

BRADSHAW, A.D. 2002. Introduction and Philosophy. Pp. 4-9. In: M.P. Perrow \& A.J. Davy (eds.). Handbook of ecological restoration - Volume 1: Principles of Restoration. Cambridge University Press. 460p.

BRADSHAW, A.D. \& CHADWICK, M.J. 1980. The restoration of land: the ecology and reclamation of derelict and degraded land. University of California Press, Los Angeles, 299 p.

BROWN, R.T.; AGEE, J.K. \& FRANKLIN, J.F. 2004. Forest restoration and fire: Principles in the context of place. Conservation Biology, 18: 903-912.

CABIN, R.J. 2007. Science-driven restoration: a square grid on a round Earth? Restoration Ecology, 15: 1-7.

CHOI, Y. D. 2007. Restoration ecology to the future: A call for new paradigm. Restoration Ecology, 15: 351-353.

CHOI, Y. D. 2004. Theories for ecological restoration in changing environment: toward "futuristic" restoration. Ecological Research, 19: 75-81.

CLEWELL, A.F. \& RIEGER, J.P. 1997. What practitioners need from restoration ecologists. Restoration Ecology, 5: 350-354.

CROSSMAN N.D.; BRYAN, B.A.; OSTENDORF, B. \& COLLINS, S. 2007. Systematic landscape restoration in the ruralurban fringe: meeting conservation planning and policy goals. Biodiversity and Conservation, 16: 3781-3802.

DAVIS, M.A. 2000. Restoration: a misnomer? Science, 287: 1203.

DORROUGH, J.; VESK, P.A. \& MOLL, J. 2008. Integrating ecological uncertainty and farm-scale economics when planning restoration. Journal of Applied Ecology, 45: 288-295. 
EHRENFELD, J.G. 2000. Defining the limits of restoration: the need for realistical goals. Restoration Ecology, 8: 2-9.

EHRENFELD, J.G. \& TOTH, L.A. 1997. Restoration ecology and the ecosystem perspective. Restoration Ecology, 5: 307-317.

ENGEL, V.L. \& PARROTTA, J.A. 2003. Definindo a restauração ecológica: tendências e perspectivas mundiais. Pp 3-25. In: P.Y. Kageyama, R.E. Oliveira, L.F. Moraes, V.L. Engel \& F.B. Gandara (orgs.). Restauração Ecológica de Ecossistemas Naturais. Ed. Fepaf, Botucatu, SP. 340p.

FATTORINI, M. \& HALLE, S. 2004. The dynamic environmental filter model: how do filtering effects change in assembling communities after disturbance? Pp. 96-114. In: V.M. Temperton, R.J. Hobbs, T. Nuttle \& S. Halle (eds.). Assembly rules and restoration ecology: bridging the gap between theory and practice. Island Press, Washington, D.C. 424p.

GEIST, C. \& GALATOWITSCH, S. M. 1999. Reciprocal model for meeting ecological and human needs in restoration projects. Conservation Biology, 13: 970- 979.

GIARDINA, C.P.; LITTON, C.M.; THAXTON, J.M.; CORDELL, S.; HADWAY, L.S. \& SANDQUIST, D.R. 2007. Science driven restoration: A candle in a demon haunted world - Response to Cabin (2007). Restoration Ecology, 15: 171-176.

GILLILAN, S.; BOYD, K.; HOITSMA, T. \& KAUFFMANN, M. 2005. Challenges in developing and implementing ecological standards for geomorphic river restoration projects: a practitioner's response to Palmer et al. (2005). Journal of Applied Ecology, 42: 223-227.

HALLE, S. 2007. Present state and future perspectives of restoration ecology - Introduction. Restoration Ecology, 15: 304-306.

HALLE, S. \& FATTORINI, M. 2004. Advances in restoration ecology: insights from aquatic and terrestrial ecosystems. Pp. 1033. In: V.M. Temperton, R.J. Hobbs, T. Nuttle \& S. Halle (eds.). Assembly rules and restoration ecology: bridging the gap between theory and practice. Island Press, Washington, D.C. 424p.

HIGGS, E. 2005.The two-culture problem: ecological restoration and the integration of knowledge Restoration Ecology, 13: 159-164.

HIGGS, E. 1997.What is good ecological restoration? Conservation Biology, 11: 338-348.

HOBBS, R.J. 2005. The future of restoration ecology: challenges and opportunities. Restoration Ecology, 13: 239-241.
HOBBS, R.J. \& NORTON, D.A. 2004. Ecological filters, thresholds, and gradients in the resistance to Ecosystem Reassembly. Pp. 73-95. In: V.M. Temperton, R.J. Hobbs, T. Nuttle \& S. Halle (eds.). Assembly rules and restoration ecology: bridging the gap between theory and practice. Island Press, Washington, D.C. 424p.

HOBBS, R.J. \& HARRIS, J.A. 2001. Restoration ecology: repairing the earth's ecosystems in the new millennium. Restoration Ecology, 9: 239-246.

HOLL, K.D. \& HOWARTH, R.B. 2000. Paying for restoration. Restoration Ecology, 8: 260-267.

HUXEL, G.R. \& HASTINGS, A. 1999. Habitat loss, fragmentation, and restoration. Restoration Ecology, 7: 309-315.

ITTO - International Tropical Timber Organization. ITTO guidelines for the restoration, management and rehabilitation of degraded and secondary tropical forests. ITTO Policy Development Series, 13, 2002, 84 p. <http:// www.itto.int/direct/ topics/topics_pdf.../topics_id $=1540000 \&$ no=1.> (Acesso em 12/09/2006).

LAKE, P. S. 2001. On the maturing of restoration: Linking ecological research and restoration. Ecological Management \& Restoration, 2: 1-3.

LAMB, D. \& GILMOUR, D. 2003. Rehabilitation and restoration of degraded forests. IUCN, n.s in Forest Conservation, 110 p. $<$ http:// www.iucn.org/dbtw-wpd/edocs/FR-IS-005.pdf > (Acesso em 12/08/2007).

LAMB, D; PARROTTA, J.; KEENAN, R. \& TUCKER, N. 1997. Rejoining habitat remnants: restoring degraded forest lands. Pp. 366-385. In: W.F. Lawrance \& R.O. Bierregaard Jr. (eds.). Tropical Forest Remnants: ecology, management and conservation of fragmented communities. University Chicago Press. 632p.

MAINA, G.G. \& HOWE, H.F. 2000. Inherent rarity in community restoration. Conservation Biology, 14: 1335-1340.

MANNING, A.D.; LINDENMEYER, B.D. \& FISCHER, J. 2006. Stretch goals and backcasting: Approaches for overcoming barriers to large-scale ecological restoration. Restoration Ecology, 14: 487-492.

MANSOURIAN, S. 2005. Overview of forest restoration strategies and terms. Pp. 8-13. In: S. Mansourian, D.Vallauri \& N. Dudley (eds.). Forest restoration in landscape: beyond planting trees. Ed. Springer, WWF. 437p. 
NUTTLE, T. 2007. Evaluation of restoration practice based on environmental filters. Restoration Ecology, 15: 330-333.

OLIVEIRA, R.E. \& ENGEL, V.L. 2009. Pesquisa e prática em restauração ecológica de ecossistemas naturais: uma análise bibliográfica. In: III Congresso Latino Americano de Ecologia, São Lourenço, Minas Gerais, Brasil. 1CD-ROM.

OLIVEIRA, R.E.; MORAES, L.F.D. \& ENGEL, V.L. 2009. Do restoration projects integrate with the landscape? Pp. 156-157. In: Latin America IALE Conference - Landscape Ecology in Latin America: challenges and perspectives. Campos do Jordão, SP, Brasil.

PALMER, M.A.; FALK, D.A. \& ZEDLER, J.B. 2006. Ecological Theory and Restoration Ecology. Pp. 1-10. In: A. Palmer \& J.B. Zedler (eds.). Foundations of restoration ecology. Island Press, Washington, $518 \mathrm{p}$.

PALMER, M.A.;AMBROSE, R.F. \& POFF, N.L. 1997. Ecological theory and community restoration ecology. Restoration Ecology, 5: 291-300.

PALMER, M.A. \& FILOSO, S. 2009. Restoration of ecosystem services for environmental markets. Science, 31: 575-576.

PARKER, T.V. 1997. The scale of successional models and restoration objectives. Restoration Ecology, 5: 301-306.

PARKER, V.T. \& PICKETT, S.T.A. 2000. Restoration as an ecosystem process: implications of the modern ecological paradigm. Pp. 17-32. In: K.M. Urbanska, N.R.Webb \& P.J. Edwards. Restoration Ecology and sustainable development. Cambridge University Press. 413p.

PASTOROK, R.A.; MACDONALD, A. \& SAMPSON, J.R. 1997. An ecological decision framework for environmental restoration projects. Ecological Engineering, 9: 89-107.

PETENON, D. \& PIVELLO, V.R. 2008. Plantas invasoras: representatividade da pesquisa dos países tropicais no contexto mundial. Natureza \& Conservação, 6: 66-77.

SCHAEFER, V. 2006. Science, stewardship, and spirituality: The human body as a model for ecological restoration. Restoration Ecology, 14: 1-3.

SCHROTT, G.R.; WITH, K.A. \& KING, A.W. 2005. Demographic limitations of the ability of habitat restoration to rescue declining populations. Conservation Biology, 19: 1181-1193.

SER - SOCIETY FOR ECOLOGICAL RESTORATION. 2004. Science \& Policy Working Group. The SER international primer on ecological restoration. < http://www.ser.org/content/ ecological_restoration_primer.asp>. (Acesso em 10/10/07).

TEMPERTON, V.M. 2007. The recent double paradigm shift in restoration ecology. Restoration Ecology, 15: 344-347.

\section{UNITED STATES ENVIRONMENTAL PROTECTION}

AGENCY, Principles for the Ecological Restoration of Aquatic Resources. $\quad<\mathrm{http}$ //www.epa.gov/owow/wetlands/restore/ principles.html> (Acesso em 12/12/2009).

URBANSKA, K.M.; WEBB, N.R. \& EDWARDS, P.J. 2000. Restoration Ecology and sustainable development. Cambridge University Press, 420 p.

VERHOEVEN, J.T.A.; SOONS, M.B.; JANSSEN, R.; OMTZIGT, M. 2008. An operational landscape unit approach for identifying key landscape connections in wetland restoration. Journal of Applied Ecology, 45: 1496-1503.

WALKER, L.R. \& DEL MORAL, R. 2003. Application of theory to rehabilitation. Pp. 283-327. In: L.R. Walker \& R. Del Moral (eds.). Primary succession and ecosystem rehabilitation. Cambridge University Press, Cambridge, UK, 445 p.

WHISENANT, S. 1999. Wildland degradation and repair. Pp.1-23. In: S. Whisenant (ed). Repairing damaged wildlands. Cambridge University Press, Cambridge, UK, 309 p.

WHITMORE, T.D. 1988. Forty years of rain forest ecology 19481988 in perspective. Geo Journal, 19: 347-360.

Submetido em 15/09/2010 Aceito em 18/04/2011 
Anexo 1. Conceitos e definições do termo "Restauração Ecológica" em diferentes momentos no tempo, desde 1980 até o presente. Appendix 1. Concepts and definitions of the term "Ecological Restoration" at different moments in time from 1980 to the present.

\section{Ano Conceito}

A restauração envolve toda e qualquer atividade que busque melhorar as condições de um sítio/local danificado, ou

1980 recriar as condições que foram destruídas ou danificadas no mesmo, tornando-o capaz de ser novamente utilizado, gerando os mesmos benefícios, de forma que seu potencial biológico seja recuperado. (Bradshaw \& Chadwick 1980, retirado de Higgs 1997) nativo, histórico e pré-definido. O objetivo desse processo é retomar a estrutura, função, diversidade e dinâmica desse ecossistema específico. (SER 1990, retirado de Higgs 1997)

A restauração ecológica de ecossistemas e paisagens degradados é uma estratégia de conservação que expande 1990 as possibilidades para proteção e melhoria da diversidade biológica na Terra. (Falk 1990, retirado de Geist \& Galatowitsch 1999).

A restauração é o retorno de um ecossistema a uma condição próxima à condição original, antes dos distúrbios...

1992 Nesse processo são recriadas a estrutura e função desse ecossistema... O objetivo é buscar/obter um sistema funcional e auto-regulado, integrado à paisagem na qual se insere. (USNRC 1992, retirado de Higgs, 1997)

Restauração sensu stricto: Ações que levam à recuperação completa de um ecossistema à sua condição pré-distúrbio (pré-degradação) em termos de estrutura e função. Restauração sensu lato: Ações que buscam reverter a degradação e direcionar a trajetória a uma direção que leve ao mais próximo das condições originais do ecossistema existente em um determinado local. (Aronson et al. 1993, retirado de Walker \& Del Moral 2003).

1994 A restauração ecológica é definida como o manejo que busca restaurar comunidades biológicas em particular, a uma condição próxima àquela que estava presente em um determinado momento, escolhido, no tempo. (Atkinson 1994)

1995 A restauração ecológica é o processo de renovação e manutenção da saúde do ecossistema-alvo. (SER 1995, retirado de Higgs 1997)

A restauração ecológica é o processo de auxiliar a recuperação e manejo da integridade ecológica do sistema. Essa integridade ecológica inclui níveis mínimos de variabilidade e biodiversidade e a ocorrência de processos e estruturas ecológicas, apresentadas num contexto histórico-regional e que envolve práticas sustentáveis. (SER 1996, retirado de Bradshaw 2002)

1997 A restauração é o processo de induzir e assistir componentes bióticos e abióticos de um ambiente para recuperar o estado em que esses existiam em seu estado original. (Bradshaw 1997, retirado de Lake 2001)

1997* A restauração tem sido contextualizada como uma intervenção sobre a dinâmica em processo de um determinado local, ou uma "sucessão aplicada". (Parker 1997)

1997 A restauração busca reverter uma área (florestal) à sua condição original presumida. (Lamb et al. 1997)

2003 A Restauração ecológica é o restabelecimento da estrutura, produtividade e diversidade de espécies (da floresta) originalmente presente. (Lamb \& Gilmour 2003)

2004 A restauração ecológica é o processo de assistência à recuperação de um ecossistema que foi degradado, danificado ou destruído. (SER 2004)

2004 A restauração ecológica envolve um grande rol de atividades relacionadas à reparação de ecossistemas danificados ou degradados. (Halle \& Fattorini 2004)

A restauração ecológica é definida como o processo de assistência à recuperação de um ecossistema que foi

2005 degradado, danificado ou destruído. É uma atividade intencional, que inicia ou acelera essa recuperação, com respeito à sua saúde, integridade e sustentabilidade (Mansourian 2005)

A restauração ecológica é o conjunto de práticas que compõem todo o campo da restauração, incluindo a base

2005 científica da ecologia da restauração, e todo o arcabouço político, tecnológico, econômico, social e cultural do envolvimento humano nesse campo. (Higgs 2005)

2009 Restauração é o retorno de um ecossistema degradado a situação muito próxima de seu potencial natural remanescente. (United States Environmental Protection Agency 2009)

2009 A restauração ecológica é uma atividade que resulta no retorno de um ecossistema a um estado não alterado. (Palmer \& Filoso 2009) 